\title{
Análisis de las diferencias de género en proyectos de vida de menores en riesgo de exclusión social
}

\author{
Garcés Delgado, Yaritza \\ Facultad de Educación, Universidad de La Laguna, San Cristóbal de La Laguna, España \\ ygarcesd@ull.edu.es \\ yaritzagd@gmail.com
}

Santana Vega, Lidia E. Facultad de Educación, Universidad de La Laguna, San Cristóbal de La Laguna, España Isantana@ull.es

\section{Resumen}

Esta comunicación presenta parte de los resultados de un estudio de mayor amplitud. El objetivo principal es analizar las diferencias de género en el proceso de configuración de los proyectos de vida de adolescentes en riesgo de exclusión social. Utilizando el método de estudio de casos múltiples e instrumentos y técnicas tanto cualitativas como cuantitativas se describen los casos de seis adolescentes en riesgo de exclusión social. Los resultados muestran diferencias de género entre los/las adolescentes a la hora de configurar sus proyectos vitales y las dificultades para planear un futuro lejos de los procesos de exclusión social en el que se han desarrollado. Las vías de estudio que se abren tras esta investigación giran en torno a cómo orientar y empoderar al colectivo en el desarrollo de estrategias y habilidades que les permitan construir un futuro óptimo para la transición a la vida activa.

\section{Abstract}

The following paper introduces part of the results of a wider and more complex research. Its main target consists of analyzing the differences based on gender in the process of configuration of the life projects of adolescents at risk of social exclusion. By using the multiple-case study approach, and both qualitative and quantitative instruments and techniques, the cases of six adolescents at risk of social exclusion are described. The results manifest significant differences based on gender among adolescents when shaping their vital projects and their difficulties to plan a future far from the social exclusion processes in which they have developed their lives. The lines of study opened after this research revolves around the procedure to guide and empower the collective in the development of strategies and skills allowing them to construct an optimal future for their transition to an active life.

Palabras clave: género, exclusión social, proyectos de vida, estudio de casos.

Keywords: gender, social exclusion, life projects, case studies.

\section{INTRODUCCIÓN}

La sociedad actual presenta una serie de barreras sociales y estructurales que dificultan la integración, adaptación y participación social de jóvenes en riesgo de exclusión social (Bendit y Hahn-Bleibtreu, 2008) y que, además, incrementan las diferencias de género en distintos ámbitos de la vida. Algunos estudios explican las «trayectorias fallidas» de estos/as jóvenes (Bautista-Cerro y Melendro, 2011; Melendro, 2011), otros trabajos demandan la restauración de las políticas sociales de atención a estos colectivos (Ballester, Caride, Melendro y Montserrat, 2016; Du Bois-Reymond y López, 2004). Los elementos sociales y personales que intervienen en el proceso de construcción del proyecto vital son determinantes para afrontar con solvencia los obstáculos a los que se enfrentan los/as jóvenes en riesgo de exclusión (Ballester, Caride, Melendro y Montserrat, 2016; Casas y Monserrat, 2009; Melendro, 2011; Melendro, Cruz, Iglesias y Montserrat, 2014).

Los/as adolescentes en situación de exclusión están en riesgo de sufrir injusticia social, desigualdad de oportunidades y desventaja respecto al grupo de iguales. Un/a adolescente en riesgo de exclusión tendrá 
menos posibilidades de elaborar un proyecto de vida atendiendo a la interacción de las elecciones personales y el contexto (Damon, 2009; Parrilla, Gallego y Moriña, 2010). Hay tres tipos de proyectos centrados en la adolescencia: el escolar, el profesional y el de vida. La configuración del proyecto escolar y profesional supone establecer metas a corto y medio plazo; posibilita la madurez cognitiva, el desarrollo de la personalidad y la consolidación de la identidad social. Sin embargo, un proyecto vital requiere de metas establecidas a largo plazo, es el estilo de vida que un/a adolescente pretende adoptar en un futuro (Boutinet, 2002).

Respecto a la configuración del proyecto vital y a las diferencias de género que puedan encontrarse entre los/as adolescentes en riesgo de exclusión social, son pocos los estudios que hasta ahora abordan el tema. Sin embargo, Rodríguez-Martínez y Blanco (2015) describen que las principales diferencias entre chicas y chicos respecto a la proyección vital se centran en la toma de decisiones en el ámbito académico y en las influencias de los/as familiares, los/as docentes y el grupo de iguales. En este sentido, las chicas [...] encuentran en la educación oportunidades para un proyecto de vida diferente (Rodríguez-Martínez y Blanco, 2015, p.66).

La configuración de un proyecto vital sano permitiría la inclusión social de estos/as adolescentes; escapar de la exclusión dependerá de la capacidad para afrontar las situaciones personales y de los apoyos, recursos, expectativas y oportunidades del entorno (Ballester, Caride, Melendro y Montserrat, 2016; Parrilla, Gallego y Moriña, 2010). Los/as profesionales de la educación deben desarrollar estrategias y modelos de integración que favorezcan la adaptación de adolescentes en riesgo, iniciándoles en el correcto tránsito a la vida adulta (Melendro, Cruz, Iglesias y Montserrat, 2014; Santana, Alonso y Feliciano, 2018) desde una perspectiva de género (Santana, Feliciano y Jiménez, 2012).

\section{MÉTODO}

En esta investigación se desarrolla un estudio de casos múltiples. Este tipo de método permite desarrollar, describir y explicar los fines de la investigación de una forma detallada y rigurosa (Stake, 2005; Yin, 2009).

\subsection{Participantes}

Se seleccionaron intencionalmente seis adolescentes. Los criterios de selección fueron: 1) estar en riesgo de exclusión social, 2) tener edades entre los 15-17 años y 3) obtener el mismo número de casos de chicas y chicos. En la Tabla 1 se especifican las características generales y específicas de los casos.

Tabla 1. Características de los participantes.

\begin{tabular}{|c|c|c|c|c|c|c|c|}
\hline \multirow{2}{*}{$\begin{array}{l}\text { Criterios } \\
\mathrm{N}^{\circ} \text { casos }\end{array}$} & \multirow{2}{*}{$\begin{array}{c}\text { Características } \\
\text { generales } \\
\text { Seis }\end{array}$} & \multicolumn{6}{|c|}{ Características particulares } \\
\hline & & Caso 1 & Caso 2 & Caso 3 & Caso 4 & Caso 5 & Caso 6 \\
\hline Sexo & equitativo & Mujer & Mujer & Hombre & Hombre & Hombre & Mujer \\
\hline Edad & 16-17 años & 17 & 17 & 17 & 17 & 16 & 16 \\
\hline $\begin{array}{c}\text { Factor de } \\
\text { riesgo }\end{array}$ & $\begin{array}{c}\text { Factores } \\
\text { Varios } \\
\left(^{*}\right)\end{array}$ & $\begin{array}{c}\text { ACAD } \\
\text { CONT FAMI }\end{array}$ & $\begin{array}{l}\text { ACAD } \\
\text { CONT }\end{array}$ & $\begin{array}{c}\text { ACAD } \\
\text { CONT } \\
\text { FAMI } \\
\text { DELI } \\
\text { DROG }\end{array}$ & $\begin{array}{l}\text { ACAD } \\
\text { CONT } \\
\text { AUTO } \\
\text { DROG }\end{array}$ & $\begin{array}{c}\text { ACAD } \\
\text { CONT FAMI } \\
\text { PERS }\end{array}$ & $\begin{array}{l}\text { ACAD } \\
\text { CONT }\end{array}$ \\
\hline Centros & $\mathrm{Si}$ & Atajo & Atajo & $\begin{array}{l}\text { Atajo } \\
\text { Tafira }\end{array}$ & Atajo & Atajo & Atajo \\
\hline
\end{tabular}

(*)ACAD: académico; FAMI: familiar; DELI: delictivo; DROG: drogadicción; CONT: contexto; PERS: personal; AUTO: autoexclusión.

El criterio de heterogeneidad de los estudios de casos múltiples se cumple con las singularidades que presenta cada adolescente. 


\subsection{Instrumentos}

Se utilizaron instrumentos de naturaleza cualitativa y cuantitativa en relación a las necesidades de la investigación. Esta combinación de técnicas proporciona mayor información que la proporcionada por un único enfoque (Flick, 2004).

Las técnicas cualitativas: 1) Entrevistas abiertas a los/as adolescentes [EA-A] sobre la situación familiar, el entorno social, el conocimiento intra e interpersonal, las metas personales de vida, las expectativas académico-laborales, etc. 2) Entrevistas abiertas a la educadora del centro [EA-E], sobre los temas abordados en la entrevista con los/as adolescentes; persiguiendo triangular la información. 3) Diario de campo [DC] para recoger información sobre las sesiones realizadas. La técnica cuantitativa: se adaptó e implementó el Cuestionario de Orientación Académica y Laboral diseñado por el GIOES de la Universidad de La Laguna. En la Tabla 2 se presentan las características del mismo.

Tabla 2. Dimensiones del COAL.

\begin{tabular}{cccc}
\hline Dimensiones & Pregunta & Ítems & $\alpha$ de Cronbach \\
\hline Información personal & $1 ; 2 ; 3$ & 3 & - \\
\hline Expectativas académico-laborales & $4 ; 5$ & 2 & - \\
\hline Habilidades personales & 7 & 17 & 5 \\
\hline Metas del proyecto de vida & $6 ; 8$ & 33 & 0.67 \\
\hline Madurez vocacional & 9 & 14 & 0.85 \\
\hline Autoeficacia vocacional & 10 & 13 & 0.81 \\
\hline Claridad del proyecto personal de vida & 11 & 4 & 0.77 \\
\hline
\end{tabular}

Se realizaron adaptaciones en las escalas: a) apoyo familiar afectivo percibido (Figuera, Dorio y Forner, 2003), b) madurez vocacional (adaptación española de Álvarez, Bisquerra, Espín y Rodríguez, 2007) y c) autoeficacia vocacional (Carbonero y Merino, 2002).

\subsection{Procedimiento}

El proceso de recogida y análisis de los datos se realizó simultáneamente a lo largo de un año. El diario de campo se redactó después cada visita al Centro de Día. El COAL fue cumplimentado individualmente; su cumplimentación osciló entre los 15 y 20 minutos. Las entrevistas fueron realizadas en días y semanas diferentes; aproximada 50 minutos con cada caso. Finalmente, se llevó a cabo la entrevista con la educadora en sesiones de 37 y 48 minutos. Los datos obtenidos por medio del COAL se analizaron con estadística descriptiva, posteriormente se analizó los datos de forma cualitativa. El análisis de las EA-A, EA-E y DC se realizó a través del Atlas-ti.

\section{RESULTADOS}

\subsection{Contextualización}

Los/as adolescentes de este estudio están inscritos/as en el Centro de Día Atajo, éste se encuentra ubicado en el polígono de La Vega de San José en el municipio de Las Palmas de Gran Canaria. Dicho Polígono se caracteriza por tener un importante número de viviendas sociales: El Centro ATAJO está dentro de un barrio que solo tiene viviendas sociales en un estado de deterioro importante situadas alrededor del Centro de Día y del Colegio de Educación de Infantil y Primaria de la zona [DC-CONT3].

ATAJO pertenece a la Fundación ADSIS. La fundación pretende con sus itinerarios formativos y preventivos favorecer al desarrollo de sociedades más justas y equilibradas. Según la educadora del 
Centro, los factores que ponen en situación de riesgo a los/as jóvenes son: El momento de la adolescencia, el idealizar a referentes que no son los recomendados, viéndolos con normalidad y sabiendo que están pendientes de un hilo, sus propias familias en muchos casos que, aunque les apoyan, han vivido esa realidad y, también, lo que han sufrido [EA-E-CONT7].

En los casos 1, 2 y 6 la situación del contexto sociofamiliar y la personalidad de las jóvenes generan consecuencias negativas en el ámbito escolar, familiar, personal y social. Respecto al caso 3, desde su infancia frecuenta diferentes centros debido a su personalidad adictiva (drogas y robos) y a los continuos conflictos familiares. Al caso 4 hay varios factores que le hacen vulnerable socialmente; desestructuración del seno familiar, el entorno en el que se desenvuelve, su grupo de iguales y el consumo de drogas. Finalmente, el caso 5 ha sido víctima de acoso escolar y familiar durante la infancia y adolescencia.

\subsection{Expectativas académico-laborales}

Las expectativas académicas de los/as adolescentes se centran en la continuación y logro de un título educativo: Programas de Cualificación Profesional Inicial, Educación Secundaria Obligatoria y el Bachillerato. Respecto a sus expectativas laborales la mayoría se inclina por un trabajo en el sector servicios. Las adolescentes parecen tener claras las metas a corto plazo. Éstas coinciden en obtener: el graduado [...] Me lo quiero sacar porque quiero trabajar y aprender algo más» [EA-A-AC/LA8]. Respecto los adolescentes, las expectativas académicas y laborales son diferentes. El caso 3, entre sus metas prioritarias destaca: Aprender a leer más rápido y a escribir mejor. [...] Tener mi graduado y sacarme un PCPI [...] [EA-A-AC/LA13]. El caso 4 y 5 pretenden realizar en el futuro Bachillerato. Según la educadora, los jóvenes tomaron decisiones equívocas en el ámbito académico: La mayoría de ellos no siguen en el instituto porque no han tomado decisiones correctas. Han sido expulsados por el propio instituto debido a sus conductas. El carácter influye negativamente en sus decisiones [EA-E-AC/LA30].

\subsection{Habilidades personales}

La mayoría presenta dificultades para reconocer sus equivocaciones y para transmitir sus ideas a otros. Todos/as admiten que sus objetivos son poco viables e irreales; manifiestan dificultades para ser constantes en lo que se proponen. Los Casos 1, 3, 4, 5 y 6 se consideran: 1) incapaces para solucionar problemas de forma asertiva; 2) sin iniciativa para desarrollar ideas propias y 3) problemas para organizar su tiempo. La mayoría señala ser incapaz de negarse ante proposiciones perjudiciales (drogas, delincuencia, etc.). El Caso 2 se percibe a sí misma como una persona sociable y consecuente con sus actos, no obstante, destaca su dificultad para admitir sus equivocaciones.

\subsection{Expectativas y apoyo familiar afectivo percibido}

La mayoría de los casos reconoce que sus familiares prefieren que acaben los estudios antes de incorporarse al ámbito laboral. Algunos/as consideran que sus expectativas académico-laborales discrepan con las de sus familias. Las adolescentes perciben apoyo de sus familiares reconociendo que: Ellos prefieren que estudie antes que trabaje. Es que [...] siempre me han dejado hacer lo que quiera [...]. Acepto sus consejos y críticas, no me queda otra. Creo que he defraudado a mi familia por la forma de llevar mis estudios [EA-A-FAM4].

Los chicos del estudio reconocen que sus familias pretenden que obtengan el graduado y posteriormente puedan elegir entre continuar estudiando o finalmente acceder al mercado laboral. Los casos 3 y 4 presentan argumentaciones similares respecto al tema: Ellos si pudieran elegir, elegirían que yo estuviese estudiando [...] Apoyan totalmente que siga estudiando y deje de hacer el "gamberro» [EA-A-FAM10]. El caso 5, presenta una situación familiar delicada, reconoce que sus familiares no lo apoyan en sus decisiones académico-laborales: La verdad es que casi todo lo que yo quiero, ellos, lo ven negativo; sobre todo mi madre [EA-A-FAM16].

\subsection{Metas del proyecto de vida}

Estos/as jóvenes consideran objetivos prioritarios el ocio y tiempo libre, adquirir dinero y mudarse del barrio en el que viven. La mayoría quiere encontrar un trabajo para tener estabilidad económica y no 
estiman prioritario realizar una carrera, casarse y estudiar en el extranjero (menos el Caso 5). Las menores coinciden en que quieren continuar sus estudios, llevarse bien con sus familias y disfrutar del ocio y el tiempo libre: Para mí es prioritario mantener buena relación con mis padres, encontrar un trabajo pronto y ganar dinero [EA-A-MET13]. Sin embargo, las tres reconocen que: Realmente, no me he propuesto ni metas [...] a corto ni a largo plazo [...] simplemente vivo el momento [EA-A-MET20].

En el caso de los chicos es prioritario formar una familia en la que ellos mantengan a sus parejas, sobretodo en el caso 3 y 5: Mi mayor ilusión es formar una familia [...] prefiero trabajar yo antes que ella para que esté con el niño y atienda la casa [EA-A-MET15]. El caso 6 plantea metas vitales como: acabar los estudios, trabajar y disfrutar del ocio y el tiempo libre [EA-A-MET16]. Un dato curioso es que el caso 5 quiere irse a: vivir al extranjero solo [EA-A-MET17].

\subsection{Madurez vocacional}

Respecto a la madurez vocacional, estos/as adolescentes consideran que no son influenciables en la elección de una futura profesión, sin embargo, todos/as admiten cambiar continuamente sus decisiones vocacionales y profesionales. Los casos 1 y 2, coinciden: No me dejo influenciar en mis expectativas académicas y profesionales. Elegiré una profesión que me guste y no una que agrade a mis padres [EA-A-MAD6]. La joven del caso 6 afirma: Tomo decisiones equivocadas continuamente; si me dicen que no lo voy a conseguir [...] directamente ni lo intento [EA-A-MAD25]. Los jóvenes del estudio explican cuáles creen que son sus problemas para alcanzar los objetivos que se proponen: Mi mayor problema es la falta de constancia en las decisiones que tomo. Me dejo influenciar por la opinión de otros. Soy poco constante en todo lo que hago y no tengo prioridades en el ámbito personal. A veces dudo en las decisiones que tomo [EA-A-MAD19].

\subsection{Autoeficacia vocacional}

La mayoría es incapaz de reconocer sus limitaciones. Estos/as adolescentes toman decisiones precipitadas y generalmente equivocadas. Además, consideran que para conseguir un trabajo la cualificación es menos importante que la suerte. En el caso de las chicas, éstas consideran que tomar decisiones es una tarea complicada y generalmente dudan sobre las decisiones que finalmente toman: Primero lo dudo, y si lo hago bien pues bien y si no pues también. Aunque si fracaso me sienta mal [EA-A-EFI6]. En el caso de los chicos, los tres reconocen que se sienten inseguros cuando toman decisiones, ocasionándoles dificultades para lograr sus metas u objetivos. Los jóvenes tienden a pensar de manera pesimista; sus pensamientos determinan y modulan negativamente sus acciones.

\subsection{Claridad del proyecto personal de vida}

La mayoría presenta dificultades para visualizar su proyecto vital y quienes consideran que lo tienen claro no han puesto los medios necesarios para alcanzar sus metas. El Caso 1 y 5 explican que tienen claro y meditado su proyecto personal de vida. Sin embargo, les falta constancia y compromiso cuando deciden avanzar hacia una meta: Si dejo a medias lo que me propongo [...] volvería a intentarlo [EA-A-CLA7]. Los casos 2, 3, 4 y 6 confiesan que planificar su proyecto vital es una tarea ardua y que en estos momentos no es de vital importancia.

\section{DISCUSIÓN Y CONCLUSIONES}

Los casos estudiados reflejan trayectorias de jóvenes en riesgo de exclusión influenciados negativamente por las características sociales, culturales, personales y del entorno. En general, los/as participantes presentan expectativas académico-laborales difusas, escasas metas autoimpuestas, dificultades para tomar decisiones, conductas conflictivas e impulsivas. Los resultados explican las dificultades de estos/as adolescentes para proyectar un futuro lejos de los procesos de exclusión (Damon, 2009) y que existen algunas diferencias de género respecto a la configuración del proyecto vital (Rodríguez-Martínez y Blanco, 2015). Las diferencias de género encontradas se acentúan en las dimensiones: autoeficacia personal, metas de vida, expectativas académico-laborales, habilidades personales, expectativas y apoyo familiar afectivo percibido. 
Para atender las necesidades de estos/as jóvenes es necesario: a) analizar el contexto en el que se desenvuelven, b) identificar los indicadores de riesgo y de protección presentes en sus realidades y c) atender a los factores intra e interpersonales (Ballester, Caride, Melendro y Montserrat, 2016; Melendro, Cruz, Iglesias y Montserrat, 2014). Es imprescindible dar visibilidad a las problemáticas presentes en la sociedad con el fin de intervenir sobre las diferencias sociales y de género. Respecto a los casos analizados, los chicos presentan un proyecto vital más perjudicial que las chicas; debiéndose a variables intrapersonales y a las redes de apoyo del entorno (Rodríguez-Martínez y Blanco, 2015; Parrilla, Gallego y Moriña, 2010).

La principal limitación del estudio es el reducido número de casos; la investigación requeriría una muestra mayor, así como un estudio longitudinal para valorar la persistencia de los resultados obtenidos pasado un periodo de tiempo. Los resultados visibilizan las diferentes perspectivas que tienen las chicas frente a los chicos de poblaciones en riesgo a la hora de plantear sus proyectos vitales. La investigación abre nuevas líneas de trabajo sobre la influencia del entono social, familiar, cultural y económico en el proceso de construcción del proyecto vital de adolescentes en riesgo de exclusión social a fin de comprender los comportamientos y las actitudes que socialmente desarrollan estos/as jóvenes en sus entornos: ¿de qué forma se puede ayudar a los/as jóvenes en riesgo de exclusión a superar las adversidades sociales y personales que les impiden desarrollar un proyecto vital consistente? ¿Cómo potenciar la capacidad para tomar decisiones teniendo en cuenta las experiencias de vida de los menores en riesgo de exclusión?

\section{REFERENCIAS BIBLIOGRÁFICAS}

Álvarez, M., Bisquerra, R., Espín, L. y Rodríguez, S. (2007). La madurez para la Carrera en la Educación Secundaria. Sevilla, España: EOS.

Ballester, L., Caride, J., Melendro, M. y Montserrat, C. (2016). Jóvenes que construyen futuros: de la exclusión a la inclusión social. Universidad de Santiago de Compostela: Servicio de Publicaciones e Intercambio Científico.

Bautista-Cerro, M. y Melendro, M. (2011). Competencias para la intervención socioeducativa con jóvenes en dificultad social. Educación XX (14), 179-200.

Bendit, R. y Hahn-Bleibtreu, M. (2008). Youth transitions: processes of social inclusion and patterns of vulnerability in a globalised world. Budrich: Opladen.

Boutinet, A. (2002). Antropologia do projeto. Porto Alegre, Brasil: Artmed.

Carbonero, M. y Merino, E. (2002). La escala de autoeficacia vocacional: desarrollo, análisis y aplicaciones del instrumento. Revista de Psicodidáctica, (14), 99-114.

Casas, F. y Monserrat, C. (2009). Sistema educativo e igualdad de oportunidades entre los jóvenes tutelados: estudios recientes en el Reino Unido. Psicothema, 21 (4), 543-547.

Damon, W. (2009). ¿O que o jovem quer da vida? Como pais e professores podem orientar e motivar os adolescentes. São Paulo, Brasil: Summus.

Du Bois-Reymond, M. y López, A. (2004). Transiciones tipo yo-yo y trayectorias fallidas: hacia las políticas integradas de transición para jóvenes europeos. Revista de Estudios de juventud, (65), 11-29.

Figuera, P., Dorio, I. y Forner, À. (2003). Las competencias académicas previas y el apoyo familiar en la transición a la universidad. Revista de Investigación Educativa, 21 (2), 349-369.

Flick, U. (2004). Introducción a la investigación cualitativa. Madrid, España: Ediciones Morata.

Melendro, M. (2011). El tránsito a la vida adulta de los jóvenes en dificultad social. Zerbitzuan, 49, pp. 93-106. Doi:10.5569/11347147.49 .08

Melendro, M., Cruz, L., Iglesias, A. y Montserrat, C. (2014). Estrategias eficaces de intervención socioeducativa con adolescentes en riesgo de exclusión. Madrid: UNED

Parrilla, Á., Gallego, C. y Moriña, A. (2010). El complicado tránsito a la vida activa de jóvenes en riesgo de exclusión: una perspectiva biográfica. Revista de Educación, (351). 211-233.

Rodríguez-Martínez, C. y Blanco, N. (2015). Diferencias de género, abandono escolar y continuidad en los estudios. Revista Iberoamericana de Educación, vol. 68, pp. 59-78.

Santana, L.E., Alonso, E. y Feliciano, L. (2018). Trayectorias laborales y competencias de empleabilidad de jóvenes nacionales e inmigrantes en riesgo de exclusión social. Revista Complutense de Educación, 29 (2), 355-369. http://dx.doi. org/10.5209/RCED.52444

Santana, L.E., Feliciano, L. y Jiménez, A. B. (2012). Toma de decisiones y Género en Bachillerato. Revista de Educación, 359, 357-387. DOI:10.4438/1988-592X-RE-2011-359-098.

Stake, R. (2005). Investigación con estudio de casos. Madrid, España: Morata.

Yin, R. (2009, $4^{\mathrm{a}}$ ed.). Case Study Research. Design and Methods. California, US: Sage. 